\title{
Cellular localization of EMMPRIN predicts prognosis of patients with operable lung adenocarcinoma independent from MMP-2 and MMP-9
}

\author{
Wulf Sienel $^{1,7}$, Bernhard Polzer ${ }^{2,7}$, Karimah Elshawi², Michael Lindner ${ }^{3}$, Alicia \\ Morresi-Hauf $^{4}$, Christian Vay ${ }^{2,5}$, Fabian Eder ${ }^{6}$, Bernward Passlick ${ }^{1}$ and Christoph A Klein ${ }^{2}$ \\ ${ }^{1}$ Department of Thoracic Surgery, University Hospital Freiburg, Freiburg, Germany; ${ }^{2}$ Department of \\ Pathology, Division of Oncogenomics, University of Regensburg, Regensburg, Germany; ${ }^{3}$ Department of \\ Surgery, Asklepios Fachkliniken München-Gauting, Gauting, Germany; ${ }^{4}$ Department of Pathology, Asklepios \\ Fachkliniken München-Gauting, Gauting, Germany; ${ }^{5}$ Department of General, Visceral, and Pediatric Surgery, \\ Heinrich-Heine University and University Hospital, Düsseldorf, Germany and ${ }^{6}$ Department of Pathology, \\ University of Regensburg, Regensburg, Germany
}

\begin{abstract}
Extracellular matrix metalloproteinase (MMP) inducer (EMMPRIN, CD147) is a multifunctional protein that has been implicated in cancer invasion and metastasis by the induction of MMPs. To address its role in primary tumors of human non-small-cell lung cancer we assessed whether EMMPRIN expression is associated with the expression of MMP-2 and MMP-9 and with patient survival. Primary tumors of 150 patients (65 adenocarcinomas, 58 squamous cell carcinomas, and 27 of other subtypes) with completely resected lung cancers were stained by immunohistochemistry. We assessed intensity, extent, and cellular localization of EMMPRIN staining and determined MMP-2 and MMP-9 expression. 145 tumors expressed EMMPRIN (strong expression in 61 tumors), which was predominantly localized at the tumor cell membranes in $102(68 \%)$ patients. We could not determine any correlation between EMMPRIN expression and MMP-2 or MMP-9 expression. The prognostic relevance of EMMPRIN was evaluated by Kaplan-Meier and multivariate Cox regression analysis in patients with adenocarcinoma $(n=57)$ and squamous cell carcinoma of the lung $(n=56)$. The median follow-up period was $\mathbf{3 6 . 0}$ months (range 4-156 months). Staining scores for EMMPRIN and MMP-2 and MMP-9 derived from staining intensities and percentages of positive cells did not predict outcome of patients. In contrast, univariate survival analysis demonstrated that membranous localization of EMMPRIN was associated with shortened survival in patients with adenocarcinoma $(P=0.03$; log-rank test), but not in squamous cell carcinoma. For the former patients, membranous EMMPRIN expression was also an independent predictor of patient survival $(P=0.04$; Cox regression analysis). The findings point to a role of EMMPRIN for the progression of adenocarcinoma of the lung that is unrelated to its function as inducer of MMPs.

Modern Pathology (2008) 21, 1130-1138; doi:10.1038/modpathol.2008.102; published online 20 June 2008
\end{abstract}

Keywords: extracellular matrix; matrix metalloproteases; lung cancer; survival analysis; prognosis

Lung cancer is still the most common cause of cancer-related death in Europe and in the United States $^{1,2}$ with non-small-cell lung cancer affecting approximately $80 \%$ of all lung cancer patients. ${ }^{1,3}$ In

Correspondence: Professor CA Klein, MD, Department of Pathology, Division of Oncogenomics, University of Regensburg, FranzJosef-Strauss-Allee 11, Regensburg 93053, Germany.

E-mail: christoph.klein@klinik.uni-regensburg.de

${ }^{7}$ These authors contributed equally to this work and share the first authorship.

Received 14 January 2008; revised 06 May 2008; accepted 07 May 2008; published online 20 June 2008 stage I, only $60-65 \%$ of non-small-cell lung cancer patients, whose tumors were completely resected, are still alive 5 years after surgery. ${ }^{4,5}$ This obvious aggressiveness is poorly understood and prompted us to search for potential molecular markers and mechanisms for early systemic spread of nonsmall-cell lung cancer.

We previously found that disseminated cancer cells from non-small-cell lung cancer can be detected in bone marrow and lymph nodes and that the detection of such cells is specifically associated with poor survival in early stage patients. Moreover, we observed that one of the most frequently 
expressed molecules on early disseminated nonsmall-cell lung cancer cells in bone marrow is EMMPRIN/CD147 (extracellular matrix metalloproteinase (MMP) inducer), ${ }^{6}$ being present on $80 \%$ of disseminated cancer cells. EMMPRIN is a transmembrane glycoprotein present on the cancer cell surface, which was found to induce expression of MMPs in fibroblasts or tumor cells. ${ }^{7}$ It was originally designated tumor collagenase-stimulating factor ${ }^{8}$ and has been renamed because of its ability to stimulate fibroblast synthesis of MMPs such as MMP-1, -2, -3, and -9..$^{7,9,10}$ The presence of EMMPRIN on the cancer cell plasma membrane not only stimulates the production of MMPs by fibroblasts but also binds MMPs on the tumor cell surface. ${ }^{11}$ Because of its role in MMP regulation, EMMPRIN is thought to facilitate invasion and metastasis by the induction and regulation of basement membrane-degrading proteases, in particular MMP-2 and MMP-9. ${ }^{10}$ However, EMMPRIN clearly is a multifunctional protein, which has also been implicated in cell adhesion, regulation of apoptosis, angiogenesis, and drug resistance. ${ }^{12}$ To address the role of EMMPRIN in the clinical progression of nonsmall-cell lung cancer patients and to evaluate whether this role is associated with induction of MMP-2 and MMP-9, we investigated the expression of EMMPRIN, MMP-2, and MMP-9 in primary tumor samples of patients with operable non-small-cell lung cancer and analyzed the impact of EMMPRIN on metastasis and prognosis.

\section{Materials and methods}

\section{Patients}

After approval by the ethical committee of the University of Munich and after written informed consent, primary tumors of 150 consecutive patients with completely resected non-small-cell lung cancer were subjected to this study. The patient cohort consisted of 65 patients with adenocarcinoma, 58 patients with squamous cell carcinoma, 15 patients with large cell carcinoma, and 12 patients with mixed histologies. Adenocarcinomas were further differentiated into tumors with and without bronchioalveolar components (mixed BAC and non-BAC, respectively $\left.{ }^{13,14}\right)$. As only five patients displayed the mixed-BAC phenotype, subgroup analysis was not performed. Surgery was performed in the Department of Thoracic Surgery, Asklepios Fachkliniken München-Gauting. The tumors were classified according to the International Union Against Cancer TNM classification. ${ }^{15}$ The preoperative staging of all patients had resulted in the diagnosis of resectable tumors (T1-T4) without evident distant metastasis (M1) or contralateral or supraclavicular lymph node involvement (N3). In general, a lobectomy or pneumonectomy with systematic mediastinal lymphadenectomy was performed. Only patients with confirmed postoperative Ro stage were further evaluated. The final study population consisted of 35 female and 115 male patients. The median age at the time of surgery was 60 years with a range of 3780 years. Patients whose primary tumors were classified as pT3 or pT4 received adjuvant percutaneous radiotherapy of the tumor bed and patients with mediastinal lymph node involvement ( $\mathrm{pN} 2$ ) received percutaneous radiotherapy of the entire mediastinum. Systemic adjuvant therapy was not performed. The median follow-up duration was 36.0 months (mean 53.8, range 4-175). Follow-up studies included physical examination, chest X-ray, and blood tests in a 3-month interval and an additional thoracic CT scan, abdominal ultrasound, and bronchoscopy in a 6-month interval. Family practitioners were contacted to obtain information about local relapse, distant metastasis, and death. Whenever possible, a relapse was confirmed at our institution and the patient was admitted for subsequent therapy.

\section{Immunohistochemical Staining of Primary Tumors}

The expression and localization of EMMPRIN, MMP-2, and MMP-9 in primary tumors was analyzed by immunohistochemistry using the labeled streptavidin-biotin (LSAB) method. Briefly, paraffin sections were dewaxed, rehydrated, and subsequently boiled for $20 \mathrm{~min}$ in $10 \mathrm{mM}$ citric acid buffer at pH 6.0 for antigen retrieval of EMMPRIN or MMP-9. Antigen retrieval of MMP-2 was achieved by incubation with $0.08 \%$ trypsin (Sigma, St Louis, $\mathrm{MO}$, USA) for $30 \mathrm{~min}$ at $25^{\circ} \mathrm{C}$. Endogenous peroxidase activity was blocked by treating the specimens with $30 \%$ hydrogen peroxide for $10 \mathrm{~min}$. Nonspecific antibody binding was prevented by a commercial blocking agent (LSAB kit; Dako Corp., Hamburg, Germany) and the sections were incubated overnight at $4^{\circ} \mathrm{C}$ with the primary antibodies. For EMMPRIN immunostaining we used the monoclonal affinity-purified mouse antibody HIM6 (BD Pharmingen, Heidelberg, Germany), which is directed against the CD147 epitope at a final concentration of $1 \mu \mathrm{g} / \mathrm{ml}$ after dilution with background reducing antibody diluent (Dako Corp.). Slides were then incubated with biotinylated anti-mouse/antirabbit secondary antibody mixture (LSAB kit; Dako Corp.) for $30 \mathrm{~min}$ at $25^{\circ} \mathrm{C}$. Peroxidase was introduced using a streptavidin conjugate (LSAB kit; Dako Corp.). Between each step of the procedure the specimens were thoroughly rinsed with $0.1 \mathrm{M}$ TrisHCl buffer ( $\mathrm{pH}$ 8.2). Peroxidase reactivity was visualized using aminoethylcarbazole (Sigma) dissolved in dimethylformamide and $0.1 \mathrm{M}$ acetate buffer ( $\mathrm{pH}$ 5.2) resulting in a red-brown staining. Finally, the sections were counterstained with hematoxylin and mounted in Kaiser's glycerol gelatin. MMP-2 and MMP-9 immunostaining was performed as described. ${ }^{16,17}$ 


\section{Evaluation of Immunohistochemical Staining}

Two independent investigators, who were unaware of the clinical data, examined the tissue sections by light microscopy. Slides with discordant results were reevaluated and a consensus was reached $(n=7)$. Slides from previous studies with immunohistochemical staining of MMP- $2^{16}$ and MMP-9 ${ }^{17}$ were available in 141 and 124 primary tumors, respectively. EMMPRIN staining intensity of tissue sections was compared with cytoblock paraffin preparations of the breast carcinoma cell line MCF-7, which served as positive control. MMP-2 and MMP-9 staining intensity within the tumor was compared with that of macrophages or bronchiolar epithelium in the sections because these cells are known to express MMP-2 and MMP-9 and therefore served as internal positive controls. Staining intensity was classified into 0 (no staining), 1 (intensity lower than positive control), 2 (intensity equal to positive control), or 3 (intensity higher than positive control). The percentage of stained tumor cells was assessed by counting 100 tumor cells within five visual fields per slide (magnification 200-fold). As 145 primary tumors were positive for EMMPRIN and 121 for MMP-9, we decided to determine expression levels for those two antigens by using the HSCORE method. ${ }^{18}$ HSCORE values were calculated using the formula HSCORE $=((I+1) \times \mathrm{PC})$, where $I$ represents staining intensity and PC the percentage of stained tumor cells. Expression levels were categorized into low (HSCORE $\leq 200$ ) and high (HSCORE $>200$ ), roughly dividing the analyzed samples in two similar-sized groups. Expression of MMP-2 was classified as either positive or negative. Cellular localization of EMMPRIN was additionally categorized into either membranous or cytoplasmic staining pattern. Although all positive tumors showed at least weak expression levels in both compartments of the cells, one staining pattern was always clearly predominant. Of note, we did not observe areas with predominant cytoplasmic and with predominant membranous patterns within the same tumor and therefore all tumors were categorized as expressing either membranous or cytoplasmic EMMPRIN. Staining specificity was assessed by analysis of negative staining controls of every tumor, in which the primary antibody had been replaced with an isotype-matched, normal rabbit or mouse nonimmune IgG.

\section{Statistical Analyses}

All statistical analyses were performed using SPSS software version 15.0 for PC (SPSS Inc., Chicago, IL, USA). The two-sided $\chi^{2}$-test was used to analyze a possible association of EMMPRIN expression and localization with clinicopathological variables, and expression of MMP-2 and MMP-9, as well as for differences between the diagnostic subtypes of non-small-cell lung cancer. For analysis of follow-up data of patients with adenocarcinoma and squamous cell carcinoma, life table curves were calculated using the Kaplan-Meier method and survival distributions were compared by log-rank statistics. The primary end point was cancer-related death, as measured from the date of surgery to death. Data of patients who were still alive and without evidence of disease at the end of the study were censored. Multivariate analysis was performed using Cox proportional hazards analysis. The threshold for statistical significance was $P<0.05$.

\section{Results}

\section{EMMPRIN Staining Score and Cellular Localization}

EMMPRIN protein was expressed with varying intensity in the cytoplasm and on the cell membrane of tumor cells, but not in the surrounding stroma cells or in normal lung parenchyma. All control slides, for which we used an isotype rabbit IgG instead of the EMMPRIN antibody, showed no immunoreactivity. The MCF-7 formalin-fixed and paraffin-embedded carcinoma cell line, which served as positive control, was stained with similar intensity in all experiments. EMMPRIN staining was observed in 145 non-small-cell lung cancers and totally absent in five primary tumors. The resulting EMMPRIN HSCORE ${ }^{18}$ values were at a median of 194.0 (range 0-400). High EMMPRIN staining scores (HSCORE > 200) were found in $61(41 \%)$ tumors and no or low expression in 89 (59\%) tumors. Furthermore, EMMPRIN was localized either predominantly cytoplasmic or membranous, with membranous EMMPRIN being observed in $102(68 \%)$ tumors while the remaining 48 (32\%) tumors displayed predominantly a cytoplasmic staining pattern (Figure 1). There was no association of EMMPRIN expression level or localization with any of the clinicopathological parameters (data not shown).

As non-small-cell lung cancer consists of heterogeneous histological types of lung cancer, we divided the patients into three groups according to the diagnostic subtype: 65 patients with adenocarcinoma of the lung, 58 patients with squamous cell carcinoma, and 27 patients with other types of nonsmall-cell lung cancer (15 large cell carcinoma and 12 mixed histologies). No significant differences between the patient cohorts of the different tumor subtypes were detected for the clinicopathological parameters. The sole exception was an expected higher percentage of female patients in the adenocarcinoma group $\left(P<0.01 ; \chi^{2}\right)$. Of interest, there was a tendency to higher EMMPRIN expression in squamous cell carcinoma $\left(P=0.06 ; \chi^{2}\right)$, of which $52 \%$ showed HSCORE values $>200$ (Table 1 ). As the group of 27 patients with other subtypes of non-small-cell lung cancer than adenocarcinoma 

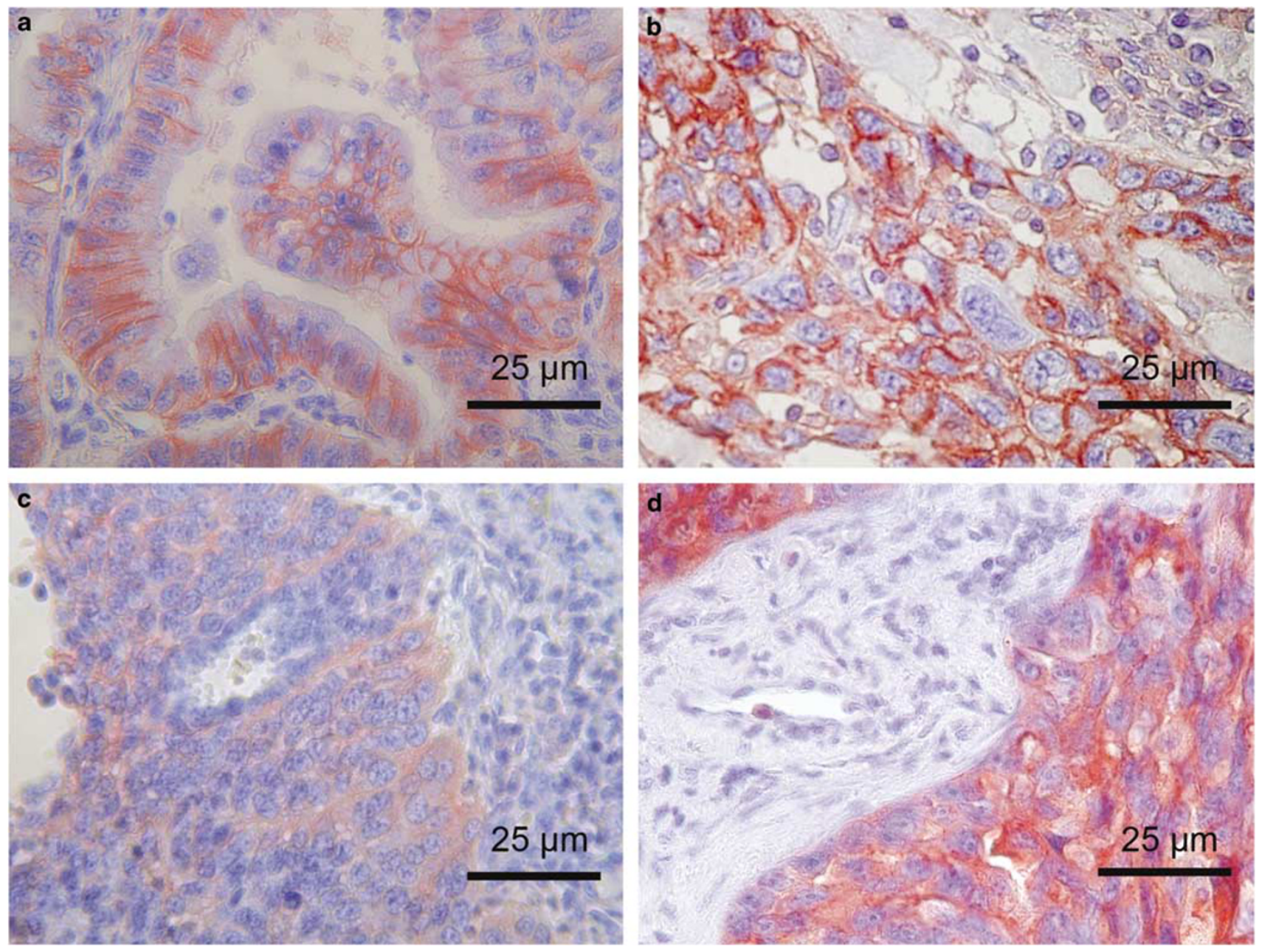

Figure 1 Immunohistochemistry of extracellular matrix metalloproteinase inducer (EMMPRIN) in primary lung adenocarcinoma. (a, b) Membranous staining pattern of EMMPRIN in primary adenocarcinoma of the lung (HSCORE $=190$ and 360, respectively). Although most cells show a weak cytoplasmic staining, a distinct membranous expression of EMMPRIN is clearly visible. (c, d) Cytoplasmic staining pattern in primary adenocarcinoma of the lung (HSCORE $=180$ and 396, respectively).

or squamous cell carcinoma consisted of heterogeneous diagnoses and rather few patients, we decided to exclude those patients from the further analyses.

Recent studies revealed that adenocarcinoma with a predominant component of bronchioalveolar carcinoma (mixed BAC) indicate a favorable prognosis for the patient compared to non-BAC adenocarcinomas. $^{13,14}$ In our study 60 of 65 patients were diagnosed with non-BAC adenocarcinomas, whereas only 5 patients showed mixed BAC histology. Most likely, due to the low number of mixedBAC cases, no impact of the two categories on survival was observed (data not shown).

\section{Association between Expression of EMMPRIN, MMP-2, and MMP-9}

Additional data on MMP-2 and MMP-9 expression were available for 141 and 124 primary tumors of our cohort, respectively. MMP-2 and MMP-9 immunoreactivity of varying intensity was only present in the cytoplasm of tumor cells, but not in the cell membrane or in the surrounding stroma. In particular, fibroblasts did not show MMP-2 or MMP-9 immunostaining. A total of 83 tumors (55\%) showed expression of MMP-2 and 121 tumors (98\%) expressed MMP-9. The median HSCORE value for MMP-9 expression was 193 (range 0-392) and 54 carcinomas were classified for high expression of MMP-9 (HSCORE > 200). We tested for a statistical association between strong MMP-2 or MMP-9 expression and EMMPRIN staining score or EMMPRIN localization, as EMMPRIN is known to induce MMPs. Surprisingly, no association between EMMPRIN HSCORE or cellular localization and the expression of MMP-2 and MMP-9 could be detected in adenocarcinoma (Table 2) or squamous cell carcinoma of the lung (Table 3). In addition, no significant association became apparent between MMP-2 or MMP-9 expression levels and diagnostic subtype, pT status, pN status, grading, age, or sex (data not shown). 
Table 1 Clinicopathological characteristics of patients and EMMPRIN expression

\begin{tabular}{|c|c|c|c|c|}
\hline \multirow[t]{2}{*}{ Variable } & \multicolumn{3}{|c|}{ Diagnostic subtype of non-small-cell lung cancer } & \multirow[t]{2}{*}{ P-value } \\
\hline & Adenocarcinoma & Squamous cell carcinoma & Miscellaneous carcinomas ${ }^{\mathrm{b}}$ & \\
\hline Total patient no. & 65 & 58 & 27 & \\
\hline \multicolumn{5}{|l|}{ Sex } \\
\hline Male & $40(62 \%)$ & $53(91 \%)$ & $22(81 \%)$ & \\
\hline Female & $25(38 \%)$ & $5(9 \%)$ & $5(19 \%)$ & $<0.01$ \\
\hline \multicolumn{5}{|l|}{ Age } \\
\hline$<60$ years & $35(54 \%)$ & $24(41 \%)$ & $15(56 \%)$ & \\
\hline$\geq 60$ years & $30(46 \%)$ & $34(59 \%)$ & $12(44 \%)$ & 0.30 \\
\hline \multicolumn{5}{|l|}{ Tumor extension } \\
\hline pT1-pT2 & $57(88 \%)$ & $43(74 \%)$ & $19(70 \%)$ & \\
\hline $\mathrm{pT} 3-\mathrm{pT} 4$ & $8(12 \%)$ & $15(26 \%)$ & $8(30 \%)$ & 0.08 \\
\hline \multicolumn{5}{|l|}{ Grading } \\
\hline G1-2 & $35(54 \%)$ & $31(54 \%)$ & & \\
\hline G3-4 & $30(46 \%)$ & $26(46 \%)$ & & 0.95 \\
\hline \multicolumn{5}{|c|}{ Lymph node status } \\
\hline pNo & $34(52 \%)$ & $27(47 \%)$ & $12(44 \%)$ & \\
\hline $\mathrm{pN} 1-\mathrm{pN} 2$ & $31(48 \%)$ & $31(53 \%)$ & $15(56 \%)$ & 0.73 \\
\hline \multicolumn{5}{|c|}{ EMMPRIN expression ${ }^{\mathrm{d}}$} \\
\hline Low & $45(69 \%)$ & $28(48 \%)$ & $16(59 \%)$ & \\
\hline High & $20(31 \%)$ & $30(52 \%)$ & $11(41 \%)$ & 0.06 \\
\hline \multicolumn{5}{|c|}{ EMMPRIN localization ${ }^{\mathrm{e}}$} \\
\hline Cytoplasmic & $26(40 \%)$ & $15(26 \%)$ & $7(26 \%)$ & \\
\hline Membranous & $39(60 \%)$ & $43(74 \%)$ & $20(74 \%)$ & 0.73 \\
\hline
\end{tabular}

EMMPRIN, extracellular MMP inducer.

aTwo-sided $P$-values were calculated by Pearson's $\chi^{2}$-test to determine a correlation between clinicopathological parameters and EMMPRIN expression level or localization.

b'Miscellaneous' represents 12 mixed histologies and 15 large cell carcinomas.

${ }^{\mathrm{C}}$ Miscellaneous carcinomas (27 cases) as well as one squamous cell carcinoma were not graded.

${ }^{d}$ EMMPRIN expression level was categorized into low (ie HSCORE $\leq 200$ ) and high (ie HSCORE $>200^{18}$ ).

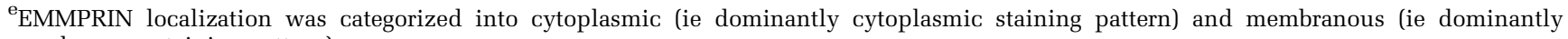
membranous staining pattern).

Table 2 EMMPRIN and MMPs in patients with adenocarcinoma

\begin{tabular}{|c|c|c|c|c|}
\hline & \multicolumn{2}{|c|}{ EMMPRIN expression $^{\mathrm{a}}$} & \multicolumn{2}{|c|}{ EMMPRIN localization $^{\mathrm{b}}$} \\
\hline & Low & High & Cytoplasmic & Membranous \\
\hline MMP-2 expression ${ }^{\mathrm{c}}$ & $n=42$ & $n=19$ & $n=24$ & $n=37$ \\
\hline No expression $(n=28)$ & $19 / 42(45 \%)$ & 9/19 (47\%) & $8 / 24(33 \%)$ & $20 / 37(54 \%)$ \\
\hline Expression $(n=33)$ & $23 / 42(55 \%)$ & $10 / 19(53 \%)$ & $16 / 24(67 \%)$ & $17 / 37(46 \%)$ \\
\hline$P$-value ${ }^{\mathrm{d}}$ & & 0.88 & & 0.11 \\
\hline MMP-9 expression ${ }^{\mathrm{a}, \mathrm{c}}$ & $n=38$ & $n=14$ & $n=24$ & $n=28$ \\
\hline Low expression $(n=34)$ & $24 / 38(63 \%)$ & $10 / 14(71 \%)$ & $14 / 24(58 \%)$ & $20 / 28(71 \%)$ \\
\hline High expression $(n=18)$ & $14 / 38(37 \%)$ & $4 / 14(29 \%)$ & $10 / 24(42 \%)$ & $8 / 28(29 \%)$ \\
\hline$P$-value ${ }^{\mathrm{d}}$ & & 0.58 & & 0.32 \\
\hline
\end{tabular}

EMMPRIN, extracellular MMP inducer; MMP, matrix metalloproteinase.

${ }^{\mathrm{a}}$ Expression levels of EMMPRIN and MMP-9 were examined using the HSCORE method. ${ }^{18}$ HSCORE values of $\leq 200$ were considered as low and values of $>200$ were considered as high concerning EMMPRIN or MMP-9 expression.

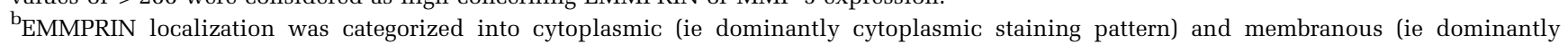
membranous staining pattern).

${ }^{\mathrm{C}}$ Data for MMP-2 expression were available in 61 and for MMP-9 expression in 52 adenocarcinomas.

${ }^{\mathrm{d}} P$-values were calculated by two-sided Pearson's $\chi^{2}$-test to determine a correlation between EMMPRIN expression level or EMMPRIN localization and expression of MMP-2 or MMP-9. 
Table 3 EMMPRIN and MMPs in patients with squamous cell carcinoma

\begin{tabular}{|c|c|c|c|c|}
\hline & \multicolumn{2}{|c|}{ EMMPRIN expression ${ }^{\mathrm{a}}$} & \multicolumn{2}{|c|}{ EMMPRIN localization $^{\mathrm{b}}$} \\
\hline & Low & High & Cytoplasmic & Membranous \\
\hline MMP-2 expression ${ }^{\mathrm{c}}$ & $n=28$ & $n=27$ & $n=15$ & $n=40$ \\
\hline No expression $(n=20)$ & $11 / 28(39 \%)$ & 9/27 (33\%) & $5 / 15(33 \%)$ & $15 / 40(37 \%)$ \\
\hline Expression $(n=35)$ & $17 / 28(61 \%)$ & $18 / 27(67 \%)$ & $10 / 15(67 \%)$ & $25 / 40(63 \%)$ \\
\hline$P$-value ${ }^{\mathrm{d}}$ & & 0.65 & & 0.78 \\
\hline MMP-9 expression ${ }^{\mathrm{a}, \mathrm{c}}$ & $n=26$ & $n=22$ & $n=15$ & $n=33$ \\
\hline Low expression $(n=22)$ & $15 / 26(58 \%)$ & $7 / 22(32 \%)$ & $7 / 15(47 \%)$ & $15 / 33(45 \%)$ \\
\hline High expression $(n=26)$ & $11 / 26(42 \%)$ & $15 / 22(68 \%)$ & $8 / 15(53 \%)$ & $18 / 33(55 \%)$ \\
\hline$P$-value ${ }^{\mathrm{d}}$ & & 0.07 & & 0.94 \\
\hline
\end{tabular}

EMMPRIN, extracellular MMP inducer; MMP, matrix metalloproteinase.

a Expression levels of EMMPRIN and MMP-9 were examined using the HSCORE method. ${ }^{18}$ HSCORE values of $\leq 200$ were considered as low and values of $>200$ were considered as high concerning EMMPRIN or MMP-9 expression.

b EMMPRIN localization was categorized into cytoplasmic (ie dominantly cytoplasmic staining pattern) and membranous (ie dominantly membranous staining pattern).

${ }^{\mathrm{C}}$ Data about MMP-2 expression were available in 55 and about MMP-9 expression in 48 squamous cell carcinomas.

${ }^{\mathrm{d}} P$-values were calculated by two-sided Pearson's $\chi^{2}$-test to determine a correlation between EMMPRIN expression level or EMMPRIN localization and expression of MMP-2 or MMP-9.

Table 4 Expression and localization EMMPRIN and cancer-free survival

\begin{tabular}{|c|c|c|c|c|}
\hline & \multicolumn{2}{|c|}{ EMMPRIN expression ${ }^{\mathrm{a}}$} & \multicolumn{2}{|c|}{ EMMPRIN localization ${ }^{\mathrm{b}}$} \\
\hline & Low & High & Cytoplasmic & Membranous \\
\hline Adenocarcinoma $^{\mathrm{c}}$ & $n=42$ & $n=14$ & $n=23$ & $n=33$ \\
\hline No cancer relapse $(n=21)$ & $16 / 42(38 \%)$ & $5 / 14(36 \%)$ & $12 / 23(52 \%)$ & $9 / 33(27 \%)$ \\
\hline Local recurrence and/or distant metastasis $(n=35)$ & $26 / 42(62 \%)$ & 9/14 (64\%) & $11 / 23(48 \%)$ & $24 / 33(73 \%)$ \\
\hline$P$-value ${ }^{\mathrm{d}}$ & & 0.60 & & 0.03 \\
\hline Squamous cell carcinoma ${ }^{\mathrm{c}}$ & $n=27$ & $n=29$ & $n=14$ & $n=42$ \\
\hline No cancer relapse $(n=24)$ & $11 / 27(41 \%)$ & $13 / 29(45 \%)$ & $7 / 14(50 \%)$ & $17 / 42(40 \%)$ \\
\hline Local recurrence and/or distant metastasis $(n=32)$ & $16 / 27(59 \%)$ & $16 / 29(55 \%)$ & $7 / 14(50 \%)$ & $25 / 42(60 \%)$ \\
\hline$P$-value ${ }^{\mathrm{d}}$ & & 0.79 & & 0.60 \\
\hline
\end{tabular}

EMMPRIN, extracellular MMP inducer.

aExression level of EMMPRIN was examined using the HSCORE method. ${ }^{18}$ HSCORE values of $\leq 200$ were considered as low and values of $>200$ were considered as high concerning EMMPRIN expression.

${ }^{b}$ EMMPRIN localization was categorized into cytoplasmic (ie dominantly cytoplasmic staining pattern) and membranous (ie dominantly membranous staining pattern).

${ }^{\mathrm{C}}$ Cancer-unrelated or perioperative death resulted in the exclusion of 8 patients with adenocarcinoma and 2 patients with squamous cell carcinoma from follow-up analyses. In one additional patient with adenocarcinoma, the time point for the development of local recurrence and distant metastasis could not be determined, resulting in 112 eligible patients (56 with adenocarcinoma and 56 with squamous cell carcinoma).

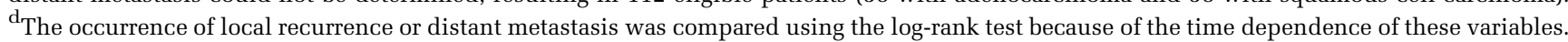

\section{Association with Recurrence, Metastasis, and Survival}

Complete follow-up was available for 57 patients with adenocarcinoma and 56 patients with squamous cell carcinoma of the lung. Eight patients with adenocarcinoma and two patients with squamous cell carcinoma had to be excluded because of death unrelated to cancer or perioperative death within 3 months after surgery. Furthermore, the time point of local recurrence or distant metastasis could not be determined for one additional patient with adenocarcinoma of the lung. The median followup duration for all 113 eligible patients was 36.0 months (range 4-156 months). Within the observation period 68 patients $(60 \%)$ relapsed either with local recurrence $(n=12)$, distant metastasis $(n=23)$, or both $(n=33)$. Interestingly, membranous EMMPRIN staining pattern was associated with local recurrence and/or distant metastasis in patients with adenocarcinoma $(P=0.03$; log-rank test), although no association could be observed for patients with squamous cell carcinoma $(P=0.60$; log-rank test). On the other hand, EMMPRIN expression score was no predictor for cancer relapse in both types of lung cancer (Table 4).

We analyzed the overall survival of the 113 eligible patients. Membranous EMMPRIN expression was strongly associated with poor survival for patients with adenocarcinoma of the lung (57 

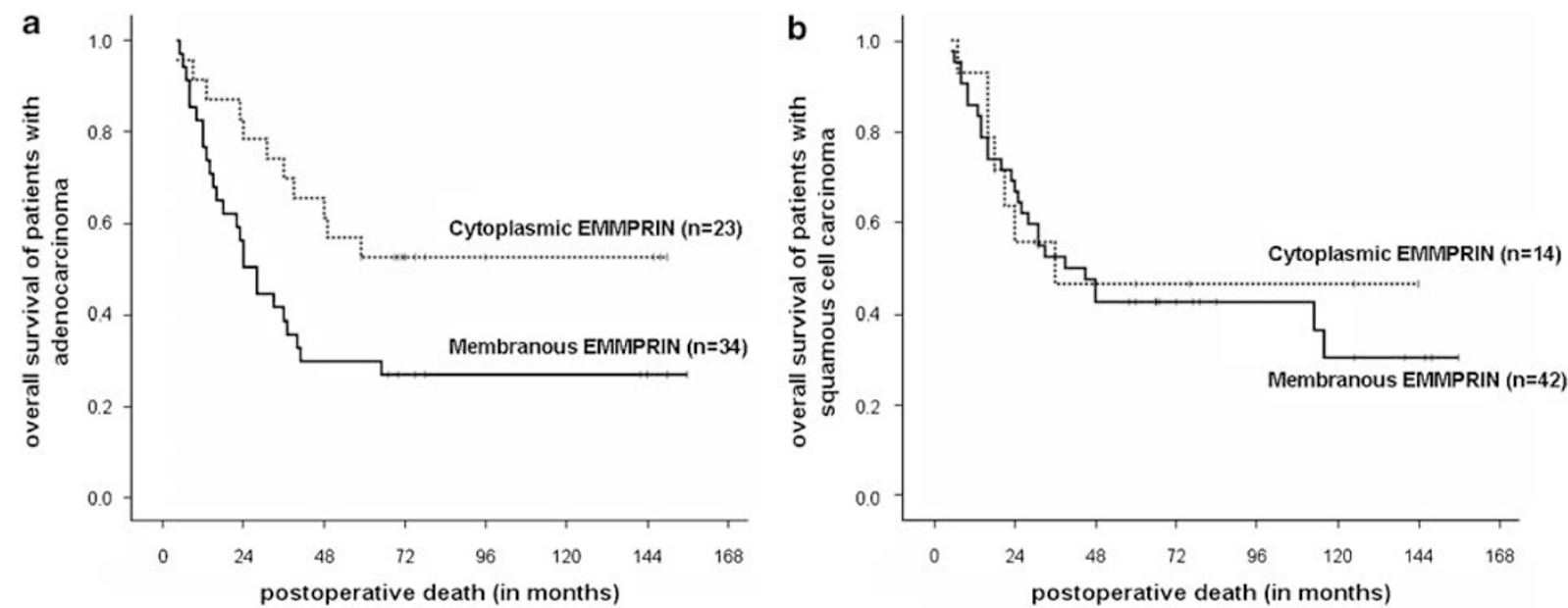

Figure 2 Cumulative cancer-related survival of patients with a membranous EMMPRIN localization compared with patients showing a cytoplasmic EMMPRIN staining pattern. (a) Analysis of 57 eligible patients with adenocarcinoma of the lung $(P=0.03$; log-rank test). (b) Survival of 56 patients with squamous cell carcinoma of the lung $(P=0.70$; log-rank test).

Table 5 Overall survival of patients with adenocarcinoma of the lung

\begin{tabular}{|c|c|c|c|c|}
\hline \multirow[t]{2}{*}{ Risk factor } & \multicolumn{2}{|c|}{ Univariate analysis ${ }^{\mathrm{a}}$} & \multicolumn{2}{|c|}{ Multivariate analysis $(n=53)^{\mathrm{b}}$} \\
\hline & Analyzed patients & $\mathrm{P}$-value & Relative risk $(95 \% \text { CI })^{\mathrm{c}}$ & P-value \\
\hline Cytoplasmic vs membranous EMMPRIN & 57 & 0.03 & $2.1(1.0-4.4)$ & 0.04 \\
\hline EMMPRIN low vs EMMPRIN high ${ }^{c}$ & 57 & 0.60 & Not included in model & \\
\hline pT1-pT2 vs pT3-pT4 & 57 & 0.17 & Not included in model & \\
\hline G1-2 vs G3-4 & 57 & 0.98 & Not included in model & \\
\hline pNo vs pN1-pN2 & 57 & $<0.01$ & $2.6(1.3-5.3)$ & 0.01 \\
\hline$<60$ vs $\geq 60$ years & 57 & 0.09 & Not included in model & \\
\hline Male vs female & 57 & 0.25 & Not included in model & \\
\hline No MMP-2 vs MMP-2 expression & 53 & 0.99 & Not included in model & \\
\hline MMP-9 low vs MMP-9 high ${ }^{\mathrm{c}}$ & 47 & 0.59 & Not analyzed & \\
\hline
\end{tabular}

EMMPRIN, extracellular MMP inducer; MMP, matrix metalloproteinase.

${ }^{\mathrm{a} C a n c e r-u n r e l a t e d ~ o r ~ p e r i o p e r a t i v e ~ d e a t h ~ r e s u l t e d ~ i n ~ t h e ~ e x c l u s i o n ~ o f ~} 8$ patients leaving $57 \mathrm{pN0}$-pN1 patients available for the analysis of possible joint effects of prognostic factors.

${ }^{\mathrm{b}}$ Step-wise multivariate analysis was performed using the Cox proportional hazard model with 53 eligible patients only, because of missing data for MMP-2 expression. No estimate of relative risk and $P$-value is given, if the variable was not significant in multivariate analysis.

${ }^{\mathrm{C}}$ Expression levels of EMMPRIN and MMP-9 were examined using the HSCORE method. ${ }^{18}$ HSCORE values of $\leq 200$ were considered as low and values of $>200$ were considered as high concerning MMP expression.

patients, $P=0.03$; log-rank test; Figure 2a), whereas the staining pattern had no influence on outcome for patients with squamous cell carcinoma (56 patients; $P=0.70$; log-rank-test; Figure 2b). As with recurrence-free survival, EMMPRIN expression HSCORE did not confer any risk for patients with either type of lung cancer (data not shown). We also performed univariate analysis for all eligible patients for tumor size, lymph node metastasis, grading, histology, age, sex, MMP-2, and MMP-9 staining. Of those, only lymph node status was an additional risk factor for overall survival of patients with adenocarcinoma $(P<0.01$; log-rank test; Table 5$)$.

To directly compare all factors for their impact on patient outcome we performed multivariate analysis (Cox regression analysis). As data for MMP-9 staining were missing for a large number of patients with adenocarcinoma and showed no association with poor survival in univariate analysis, we decided to exclude it from analysis. On the other hand, data on MMP-2 expression were available for 53 of the 57 eligible patients with adenocarcinoma of the lung and were thus included in Cox regression analysis. Adjuvant treatment did not represent a confounding variable because only patients with large tumors (pT3 and pT4) had received adjuvant radiotherapy, a fact that was already taken into account by including tumor extension into the multivariate analysis. Multivariate analysis of 53 patients revealed that EMMPRIN localization is an independent risk factor for patients with adenocarcinoma of the lung $(P=0.04$; Table 5) imposing a 2.1-fold risk for cancer-related death, with lymph node status being the only additional factor associated with decreased survival $(P=0.01$; Table 5$)$. MMP-2 expression was not associated with poor 
outcome, neither was tumor size, histology, or EMMPRIN score.

\section{Discussion}

Here we describe that EMMPRIN is an independent risk factor in lung adenocarcinoma but not in squamous cell carcinoma. Our results suggest a role of EMMPRIN in adenocarcinoma progression that is-contrary to our expectation-most likely unrelated to the induction of MMP-2 and MMP-9. Interestingly, survival of patients with adenocarcinoma was not dependent on high EMMPRIN expression scores but on membranous staining pattern. Moreover, upon multivariate analysis, membrane-bound EMMPRIN was the second strongest indicator of poor patient survival independent from lymph node status, tumor size, grading, sex, patient age, or MMP-2 expression.

A very recent study reported that EMMPRIN has no influence on survival of patients with adenocarcinoma or squamous cell carcinoma of the lung. ${ }^{19}$ Although the authors noted that EMMPRIN is localized in different cellular compartments, they did not analyze a possible association between patient outcome and localization of EMMPRIN. Likewise, mRNA expression levels would not have identified patients with poor survival-similar to an EMMPRIN staining score-as they provide no information about the actual spatial distribution of the EMMPRIN protein in cancer cells. Therefore, the prognostic significance of membrane-bound EMMPRIN would have escaped detection by microarray analyses that are currently widely used to identify prognostic markers. The prognostic impact of membranous EMMPRIN has also been observed in patients with laryngeal cancer, although for this type of cancer membranous EMMPRIN was not an independent factor for patient outcome. ${ }^{22}$ For other types of cancer, such as hepatocellular carcinoma ${ }^{23}$ and breast cancer, ${ }^{24}$ EMMPRIN expression levels were of prognostic significance, but unfortunately EMMPRIN localization and its prognostic influence were not investigated.

Survival of the patients in this study was not dependent on MMP-2 or MMP-9 expression levels, in contrast to our own previous observations. ${ }^{16,17}$ Most likely this is due to the fact that only a subgroup of the original patient cohort could be tested for EMMPRIN expression here. Within the MMP-regulating paradigm of EMMPRIN function, it is possible that membrane-bound EMMPRIN could target MMPs to the tumor cell surface ${ }^{11}$ and thereby facilitate the invasion-associated function of MMPs. $^{7,9,20}$ This hypothesis, however, does not explain why expression of MMP-2 and MMP-9 was not associated with decreased survival particularly because we were unable to differentiate between cytoplasmic and membranous MMP-2 staining patterns. Overall, we are currently unable to explain the specific mechanism underlying the association of membranous EMMPRIN and poor survival, but we deem it likely that other functions of membranous EMMPRIN are responsible for its importance in lung adenocarcinoma progression.

One possible explanation could be that a membranous location of EMMPRIN depends on monocarboxylate transporters (MCTs). MCTs have functions in the glycolytic switch occurring once cancers become hypoxic. It was shown that although knockdown of EMMPRIN by siRNA led to the accumulation of MCT4 in endolysosomal vesicles, knockdown of MCT4 caused the accumulation of EMMPRIN in the endoplasmic reticulum and decreased invasiveness in a breast cancer cell line. ${ }^{21}$ Thus, deregulation of MCTs may be involved in the cytoplasmic retention of EMMPRIN. Other candidate mechanisms of EMMPRIN-induced tumor progression are the recently described role of EMMPRIN as modulator of cellular architecture by integrin interactions that could affect tumor cell migration, ${ }^{25}$ or the induction of vascular endothelial growth factor (VEGF) by surface-bound EMMPRIN, one of the most potent angiogenetic factors. ${ }^{26,27}$ If the induction of VEGF by membranous EMMPRIN is indeed the underlying mechanism, VEGF-targeting therapies may eventually be beneficial for this subgroup of patients. Whatever the reason, among the risk factors tested in our cohort, membranous EMMPRIN was a strong independent predictor of poor outcome, which may help to identify patients with particularly aggressive disease.

\section{Acknowledgements}

We thank Manfred Meyer for excellent technical assistance. This work was supported by grants from the DFG KL 1233/1-1, SFB 469, a grant from the Bavarian State Ministry of Sciences, Research and the Arts, and the BioFuture Grant 0311884.

\section{Disclosure/conflict of interests}

The authors declared no conflict of interest.

\section{References}

1 Janssen-Heijnen ML, Gatta G, Forman D, et al. Variation in survival of patients with lung cancer in Europe, 1985-1989. Eur J Cancer 1998;34:2191-2196.

2 Jemal A, Tiwari RC, Murray T, et al. Cancer statistics, 2004. CA Cancer J Clin 2004;54:8-29.

3 el-Torky M, el-Zeky F, Hall JC. Significant changes in the distribution of histologic types of lung cancer. A review of 4928 cases. Cancer 1990;65:2361-2367.

4 Arriagada R, Bergman B, Dunant A, et al. Cisplatin-based adjuvant chemotherapy in patients with completely resected non-small-cell lung cancer. N Engl J Med 2004;350:351-360. 
5 Martini N, Bains MS, Burt ME, et al. Incidence of local recurrence and second primary tumors in resected stage I lung cancer. J Thorac Cardiovasc Surg 1995;109:120-129.

6 Klein CA, Seidl S, Petat-Dutter K, et al. Combined transcriptome and genome analysis of single micrometastatic cells. Nat Biotechnol 2002;20:387-392.

7 Biswas C, Zhang Y, DeCastro R, et al. The human tumor cell-derived collagenase stimulatory factor (renamed EMMPRIN) is a member of the immunoglobulin superfamily. Cancer Res 1995;55:434-439.

8 Prescott J, Troccoli N, Biswas C. Coordinate increase in collagenase mRNA and enzyme levels in human fibroblasts treated with the tumor cell factor, TCSF. Biochem Int 1989;19:257-266.

9 Davidson B, Goldberg I, Berner A, et al. EMMPRIN (extracellular matrix metalloproteinase inducer) is a novel marker of poor outcome in serous ovarian carcinoma. Clin Exp Metastasis 2003;20:161-169.

10 Zucker S, Hymowitz M, Rollo EE, et al. Tumorigenic potential of extracellular matrix metalloproteinase inducer. Am J Pathol 2001;158:1921-1928.

11 Guo H, Li R, Zucker S, et al. EMMPRIN (CD147), an inducer of matrix metalloproteinase synthesis, also binds interstitial collagenase to the tumor cell surface. Cancer Res 2000;60:888-891.

12 Yang JM, $\mathrm{Xu} \mathrm{Z}$, Wu $\mathrm{H}$, et al. Overexpression of extracellular matrix metalloproteinase inducer in multidrug resistant cancer cells. Mol Cancer Res 2003;1:420-427.

13 Sakao Y, Miyamoto H, Sakuraba M, et al. Prognostic significance of a histologic subtype in small adenocarcinoma of the lung: the impact of nonbronchioloalveolar carcinoma components. Ann Thorac Surg 2007;83:209-214.

14 Sakurai H, Dobashi Y, Mizutani E, et al. Bronchioloalveolar carcinoma of the lung 3 centimeters or less in diameter: a prognostic assessment. Ann Thorac Surg 2004;78:1728-1733.

15 Mountain CF. Revisions in the international system for staging lung cancer. Chest 1997;111:1710-1717.

16 Passlick B, Sienel W, Seen-Hibler R, et al. Overexpression of matrix metalloproteinase 2 predicts unfavorable outcome in early-stage non-small cell lung cancer. Clin Cancer Res 2000;6:3944-3948.

17 Sienel W, Hellers J, Morresi-Hauf A, et al. Prognostic impact of matrix metalloproteinase-9 in operable non-small cell lung cancer. Int J Cancer 2003;103: 647-651.

18 McCarty Jr KS, Miller LS, Cox EB, et al. Estrogen receptor analyses. Correlation of biochemical and immunohistochemical methods using monoclonal antireceptor antibodies. Arch Pathol Lab Med 1985;109:716-721.

19 Hakuma N, Betsuyaku T, Kinoshita I, et al. High incidence of extracellular matrix metalloproteinase inducer expression in non-small cell lung cancers. Association with clinicopathological parameters. Oncology 2007;72:197-204.

20 Tang Y, Kesavan P, Nakada MT, et al. Tumor-stroma interaction: positive feedback regulation of extracellular matrix metalloproteinase inducer (EMMPRIN) expression and matrix metalloproteinase-dependent generation of soluble EMMPRIN. Mol Cancer Res 2004;2:73-80.

21 Gallagher SM, Castorino JJ, Wang D, et al. Monocarboxylate transporter 4 regulates maturation and trafficking of CD147 to the plasma membrane in the metastatic breast cancer cell line MDA-MB-231. Cancer Res 2007;67:4182-4189.

22 Rosenthal EL, Shreenivas S, Peters GE, et al. Expression of extracellular matrix metalloprotease inducer in laryngeal squamous cell carcinoma. Laryngoscope 2003;113:1406-1410.

23 Li HG, Xie DR, Shen XM, et al. Clinicopathological significance of expression of paxillin, syndecan-1 and EMMPRIN in hepatocellular carcinoma. World J Gastroenterol 2005;11:1445-1451.

24 Reimers N, Zafrakas K, Assmann V, et al. Expression of extracellular matrix metalloproteases inducer on micrometastatic and primary mammary carcinoma cells. Clin Cancer Res 2004;10:3422-3428.

25 Curtin KD, Meinertzhagen IA, Wyman RJ. Basigin (EMMPRIN/CD147) interacts with integrin to affect cellular architecture. J Cell Sci 2005;118:2649-2660.

26 Chen X, Lin J, Kanekura T, et al. A small interfering CD147-targeting RNA inhibited the proliferation, invasiveness, and metastatic activity of malignant melanoma. Cancer Res 2006;66:11323-11330.

27 Tang Y, Nakada MT, Kesavan P, et al. Extracellular matrix metalloproteinase inducer stimulates tumor angiogenesis by elevating vascular endothelial cell growth factor and matrix metalloproteinases. Cancer Res 2005;65:3193-3199. 